\title{
XILOTECAS, IMPORTANTES COLECCIONES DE REFERENCIA
}

\section{Xylotheques, important reference collections}

\author{
Ángela María Vásquez-Correa ${ }^{1}$
}

Vásquez-Correa, A.M. (2017). Xilotecas, importantes colecciones de referencia. Colombia Forestal, 20(2), 192-201

Recepción: 29 de abril 2016

\section{Resumen}

Esta breve reseña sobre las xilotecas, como importantes colecciones de referencia, tiene como objetivo plantear el verdadero propósito y particularidades de estas colecciones, su configuración y riguroso proceso de recopilación, registro, curaduría y conservación de ejemplares, además de su importancia como legado cultural y científico de la humanidad. Presenta un poco de la historia y situación de las xilotecas en el panorama mundial y nacional y propone un análisis documentado de sus beneficios y pertinencia, que pretende incentivar a la conformación de otras colecciones y generar un cambio en su errada concepción y en su olvidada y pobre visión, así, como de los investigadores asociados a las mismas. Analiza sus necesidades inmediatas y más urgentes en Colombia, con un enfoque que pretende asegurar su apoyo y fortalecimiento por parte de las instituciones relacionadas, el desarrollo conjunto de estrategias de adquisición de ejemplares en estrecha conexión con botánicos y herbarios y la realización de investigaciones que apoyen el avance de políticas de conservación de nuestros bosques, al permitir la identificación de las especies que se transportan y comercializan, para contribuir a combatir su tala ilegal y mejorar el uso de este valioso material en el territorio nacional.

Palabras clave: anatomía de maderas, colección de referencia, conservación, tala ilegal.
Aprobación: 22 de mayo 2017

\begin{abstract}
This brief review of the xylotheque as important reference collection aims to bring the true purpose and characteristics of these collections, its configuration and rigorous collection, recording, curation and preservation of specimens, as well as its importance cultural and scientific heritage of humanity. It has a bit of history and situation of xylotheques in the world and national scene and proposes a documented analysis of the benefits and relevance, which aims to encourage the creation of other collections and generate a change in their wrong conception and its forgotten and poor vision of these and the researchers associated with them. Analyzes its immediate and most urgent needs in Colombia, with an approach that aims to ensure and strengthen their support by related institutions, the joint development of specimens acquisition strategies closely with botanical and herbariums and conducting research to support the advancement of conservation policies of our forests, to enable identification of the species being transported and marketed, to help combat illegal logging and improve the use of this valuable material in the country.
\end{abstract}

Keywords: wood anatomy, reference collection, conservation, illegal logging.

1 Grupo de Investigación Producción Ecoamigable de Cultivos Tropicales (Proecut). Departamento de Ciencias Forestales, Universidad Nacional de Colombia sede Medellín. Medellín, Colombia. amvasque@unal.edu.co 


\section{INTRODUCCIÓN}

La palabra xiloteca proviene de las raíces griegas

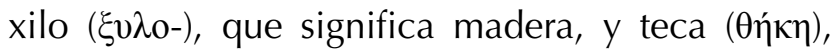
que significa caja, "lugar en que se guarda algo". Este designa un tipo de colección especial, dado que los ejemplares que la conforman siempre deben colectarse acompañados de material de herbario de respaldo, consistente en hojas y en lo posible flores y frutos del mismo árbol, lo que garantiza su identificación certera y hace posible su posterior utilización como patrones de identificación. El principal objetivo de estas colecciones es disponer de una selección de muestras de maderas que sirvan como base para la realización de estudios científicos con diferentes aplicaciones. Aunque también tienen como meta servir como material de referencia para comparaciones (Hoadley, 1990).

El término xiloteca se usa en lugar de colección de maderas con el objeto de realizar una separación conceptual entre colecciones informales y colecciones asociadas a instituciones académicas y formalmente organizadas, que además cuentan con muestrarios de placas microscópicas permanentes y registro de muestras de herbario (Lamb \& Curtis, 2005). Cabe anotar que el porcentaje de muestras de madera asociadas con registros de muestras de herbario varían mucho entre xilotecas, pues las mismas solo se convirtieron en una práctica habitual desde las dos últimas décadas del siglo XX. Las muestras más antiguas eran generalmente obtenidas de departamentos forestales o arboretos que garantizaban la perfecta identificación de los árboles (Wiedenhoeft, 2014).

El presente escrito tiene como objetivos aclarar la equivocada concepción que se tiene acerca de estas colecciones de referencia en el país, al plantear su verdadero propósito y particularidades, y su diferencia crucial con otras colecciones de maderas, así como su importancia, beneficios y pertinencia. $Y$ adicionalmente, presentar su historia y situación en el panorama mundial y nacional, además de proponer un análisis de sus necesidades inmediatas y más apremiantes en Colombia.

\section{XILOTECAS EN EL MUNDO}

Las colecciones más antiguas y que aún existen fueron establecidas en Alemania, la primera en 1770 en el Instituto de Historia e Investigación Natural (BHUw), en Berlín, y la segunda en 1817 en el Museo de Historia Natural (FRw), en Frankfort. A estas le siguen las xilotecas del Instituto de Investigaciones Forestales (DDw) en Dehradun, India, creada en 1836, la colección del Jardín Botánico Real (Kw) fundada en 1847 en Inglaterra, y las xilotecas alemanas del Jardín y Museo Botánico (Bw) en Dahlem y del Jardín Botánico de la Universidad Técnica de Dresden en Tharandt (TSFw), inauguradas en 1850 y 1852 respectivamente. En América, las colecciones más antiguas se encuentran en Estados Unidos y fueron creadas en 1889 en el Departamento de Botánica en Honolulu (BISHw) y en 1890 en el Centro Forestal Mundial en Portland (NYJw) (Lynch \& Gasson, 2010; Cornish et al., 2014).

Posteriormente, la gran mayoría de las xilotecas fueron establecidas a comienzos o mediados del siglo $X X$, su primer recuento a nivel mundial fue publicado en 1957 con el nombre de Guide to Institucional Wood Collections, donde se cuenta un total de 66 colecciones (Stern, 1957). Luego, a partir de 1988, comenzó a utilizarse el nombre de Index Xylariorum (Stern, 1988; León, 2009) y el número de xilotecas había aumentado a 134.

Más recientemente, el Index Xylariorum 4 (Lynch \& Gasson, 2010) presentó el registro de 158 xilotecas pertenecientes a 47 países, de las cuales 63 se encuentran en América (38\%), así: 21 en Norte América (tres en Canadá, 18 en Estados Unidos); siete en Centro América (tres en Costa Rica, tres en México, una en Nicaragua); seis en las islas del Caribe (dos en Cuba, dos en República Dominicana, una en Haití, una en Jamaica) y 33 en Sur América (tres en Argentina, 20 en Brasil, dos en Chile, dos en Colombia, una en Ecuador, una en Guyana, una en Perú, una en Surinam, dos en Venezuela).

El aumento en el número y tamaño de las colecciones también ha estado acompañado por la fusión de algunas de las más antiguas, lo que ha 
dado como resultado la existencia de dos grandes xilotecas a nivel mundial, cada una con 105000 ejemplares aproximadamente. Estas son: la xiloteca del Herbario Nacional de la Universidad de Leiden (Lw), en los Países Bajos, fundada en 1955, y en América, la xiloteca del Laboratorio de Productos Forestales en Madison (MADw), Estados Unidos, establecida en 1970 la cual alberga entre sus colecciones más importantes dos procedentes de Colombia y realizadas por los botánicos José Cuatrecasas y Armando Dugand. De igual manera, pueden mencionarse las colecciones del Museo Real de África Central (Tw) en Tervuren, Bélgica, fundada en 1898 con 61000 ejemplares, y las existentes en el Instituto Smithsonian (USw) iniciada en 1915 y en la Universidad de Nueva York (BWCw) en Syracuse, abierta en 1925, cada una con 40000 especímenes (Lynch \& Gasson, 2010).

Para Colombia el Index Xylariorum 4 (Lynch \& Gasson, 2010) reporta dos colecciones, la más antigua creada en 1963 en el Instituto de Investigaciones Forestales y Madereras de la Universidad Distrital Francisco José de Caldas (BOFw), en Bogotá, con 1400 muestras, y la colección del Laboratorio de Productos Forestales Héctor Anaya López (MEDELw) de la Universidad Nacional de
Colombia Sede Medellín, fundada hace 40 años, en 1975 cuando el profesor Luis Carlos Mejía Mesa comenzó un proyecto de intercambio con numerosas xilotecas en todo el mundo y desarrolló investigaciones que permitieron la colecta de material en Colombia (figura 1).

Desde 1988, esta última colección se encuentra registrada en el Index Xilariorum con el acrónimo MEDELw, y desde 2002 en el Registro Nacional de Colecciones Biológicas (X-UNCM, Registro 123) que tiene a su cargo el Instituto Alexander von Humboldt y aparece como la mayor en Colombia (figura 2). Actualmente, cuenta con 3228 ejemplares representados por 1362 especies, 857 géneros y 131 familias, con el grupo de las leguminosas (15\%) y las familias Pinaceae (6\%), Lauraceae (4\%) y Meliaceae $(4 \%)$, como las más representativas. Incluye maderas procedentes de los cinco continentes, principalmente de América (52\%), Asia (17\%), África (13\%) y Europa (10\%), y representa principalmente países como Colombia (17\%), Venezuela (9\%), Brasil (7\%), Estados Unidos (4\%), India (3\%) y Perú $(3 \%)$, sin dejar de mencionar a Alemania, Angola, Austria, Canadá, China, Costa Rica, Cuba, Inglaterra, Indonesia, Japón y Pakistán, con un porcentaje también representativo.

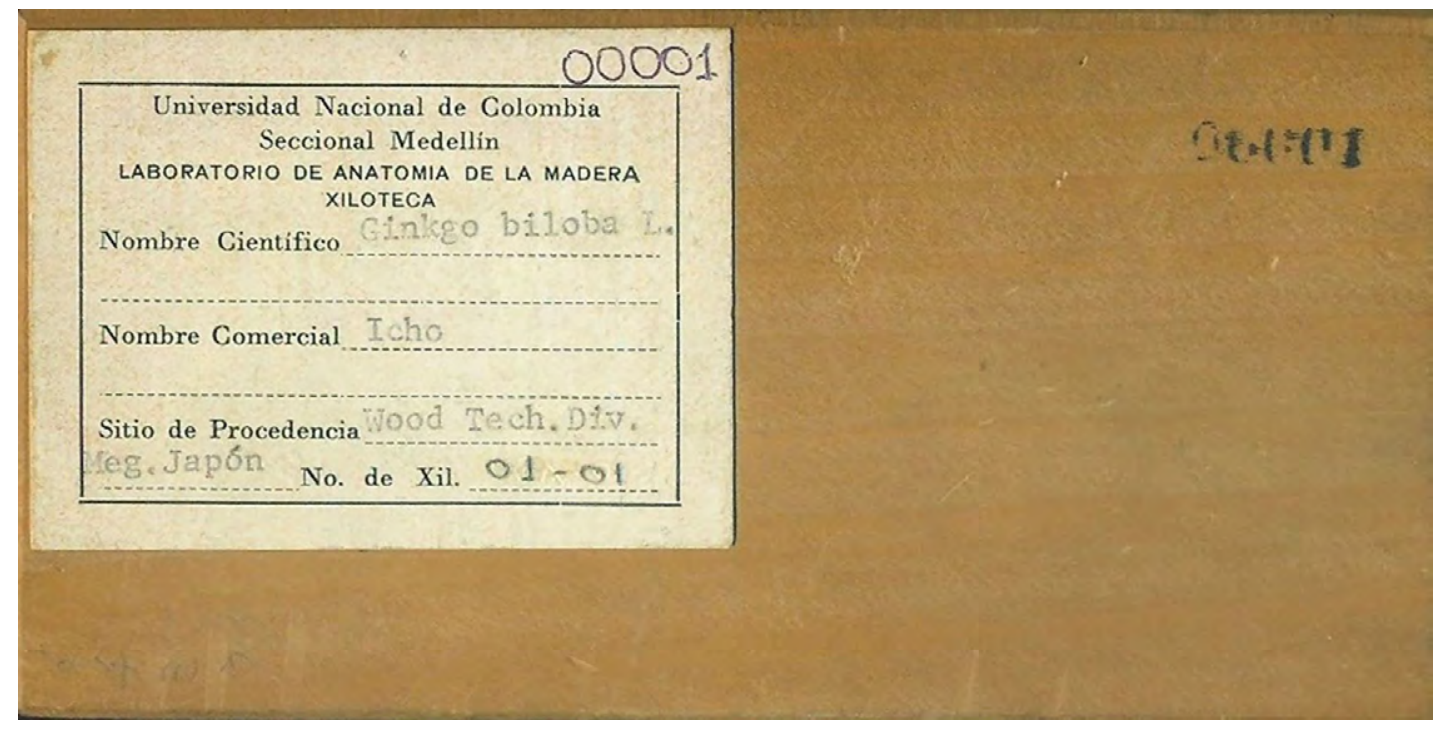

Figura 1. Primera muestra de madera que ingresó a la xiloteca en 1975, especie Ginkgo biloba L. 


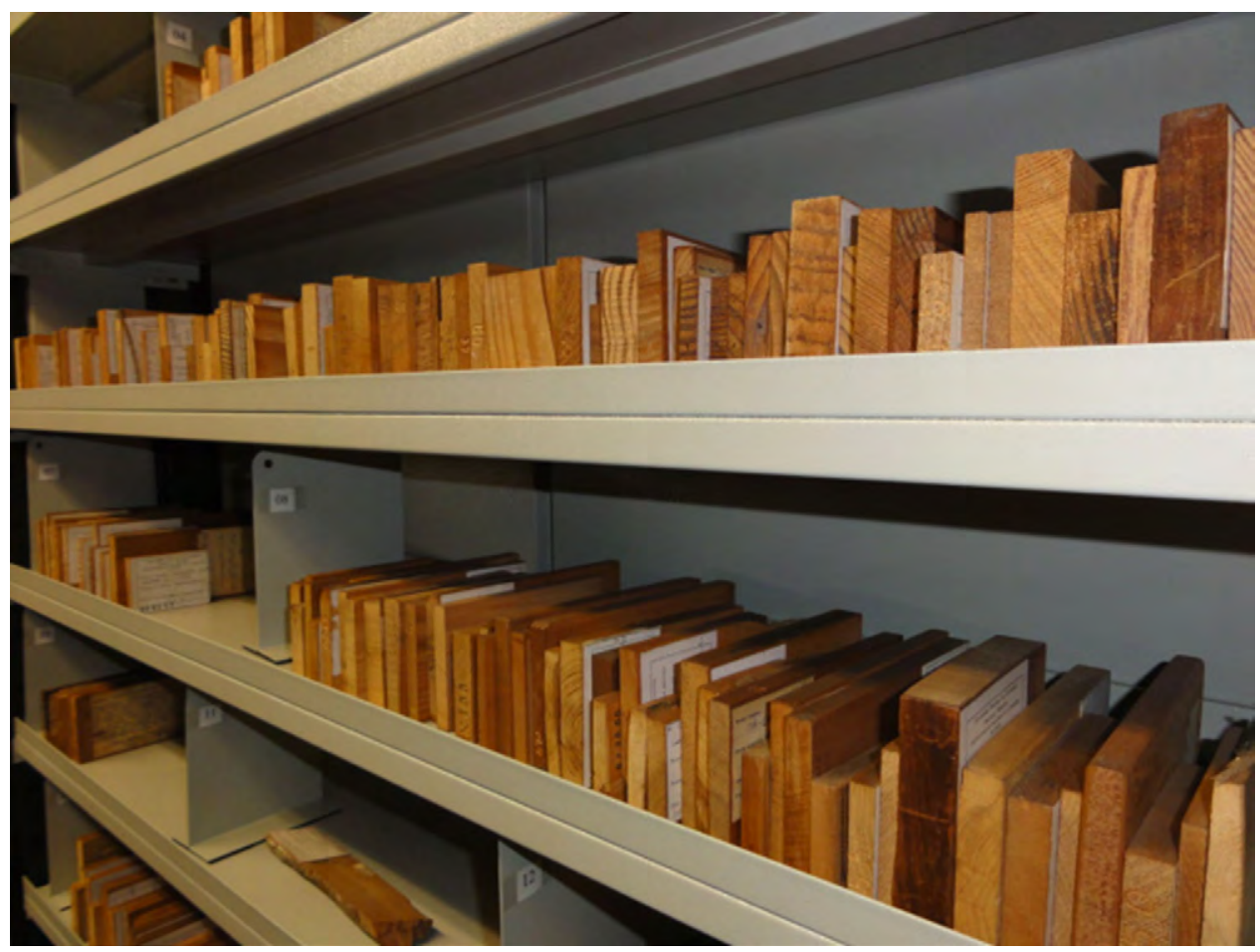

Figura 2. Xiloteca MEDELw de la Universidad Nacional de Colombia sede Medellín.

Entre las instituciones que han contribuido de manera más significativa a enriquecer esta colección es preciso mencionar que un porcentaje importante de las muestras ha ingresado a través de trabajos de investigación realizados en el Laboratorio de Productos Forestales de la Universidad Nacional de Colombia sede Medellín (9\%), seguido por muestras donadas de las xilotecas de la Universidad de los Andes (MERw), Venezuela (8\%), y del Laboratorio de Productos Forestales en Madison (MADw), Estados Unidos (7\%). Así mismo, las xilotecas de la Universidad Distrital (BOFw) en Bogotá, la del Dr. Calvino Mainieri en el Instituto de Pesquisas Tecnológicas IPT (BCTw) en São Paulo y la de la Universidad de Stellenbosch (STEw) en Sudáfrica, han aportado cada una un 3\% de los ejemplares.

Al igual que Cornish et al. (2014), se considera importante realizar este breve recuento sobre cómo se encuentra conformada la colección y cuál ha sido el patrón de adquisición de sus muestras, pues conduce a una mejor comprensión de sus procedencias y a una mayor confiabilidad en la calidad de su identificación, además de conservar la historia de la institución que la alberga.

\section{COLECCIÓN, REGISTRO, CURADURIA Y CONSERVACIÓN DE EJEMPLARES}

Se contempla que, para la obtención en campo de muestras de madera que puedan hacer parte de una xiloteca, se haga una adecuada recolección del material botánico del árbol correspondiente, siguiendo los protocolos establecidos.

Inicialmente, las xilotecas a nivel mundial contenían un gran porcentaje de muestras en formato de pequeñas tabletas de aproximadamente $6 \times 10$ $x 2 \mathrm{~cm}$, que aunque daban a las colecciones una mayor uniformidad, muchas veces no representaban de manera completa la madera de las especies, pues no era posible observar en las mismas las 
características y apariencia de la albura (parte externa) y del duramen (parte interna del tallo). Con el paso del tiempo, la creciente dificultad y costo que supone la toma de muestras de madera en campo y su correspondiente traslado, además de que en muchos casos no es posible realizar el apeo de los árboles, las actuales colecciones poseen material de muy diversas dimensiones, con muestras en forma de cuña obtenidas del árbol en pie con la ayuda de una motosierra o un formón y sierra manual, y también muestras obtenidas con barreno, aunque no sean estas las más indicadas (figura 3).

Cuando el árbol puede o debe ser apeado, se recomienda colectar una sección transversal o disco de madera de varios centímetros de espesor en la base del tallo o a la altura del pecho $(1.30 \mathrm{~m})$, que luego pueda ser fraccionada en muestras en forma de cuña que abarquen toda la longitud del radio (medula a corteza) y representen de manera más completa los cambios en color y estructura morfológica de las especies, reteniendo en algunos casos la corteza para diversos fines e intereses.
Lo ideal, y también lo acostumbrado durante los siglos IX y XX, era colectar las muestras del tallo principal del árbol seleccionado (especificando la altura en el mismo), pero la dificultad de acceso, transporte y costo de obtención de las maderas han llevado a que muchos ejemplares sean colectados de pequeñas ramas e incluso de la raíz de los individuos. En todos los casos es de suma importancia consignar información acerca de la parte del árbol de la cual proviene la muestra. Tampoco existen criterios acerca del número de árboles a ser muestreados, pues esto estará determinado en función de las posibilidades y requerimientos. Deseable es que a la xiloteca puedan ingresar mínimo dos muestras del espécimen colectado y que adicionalmente queden algunas para intercambio, que puedan ser enviadas a otras colecciones y que garanticen que dicho material no se perderá de manera definitiva en caso de alguna eventualidad o siniestro en una colección en particular.

La marcación de las muestras durante el periodo de obtención y trasporte reviste especial

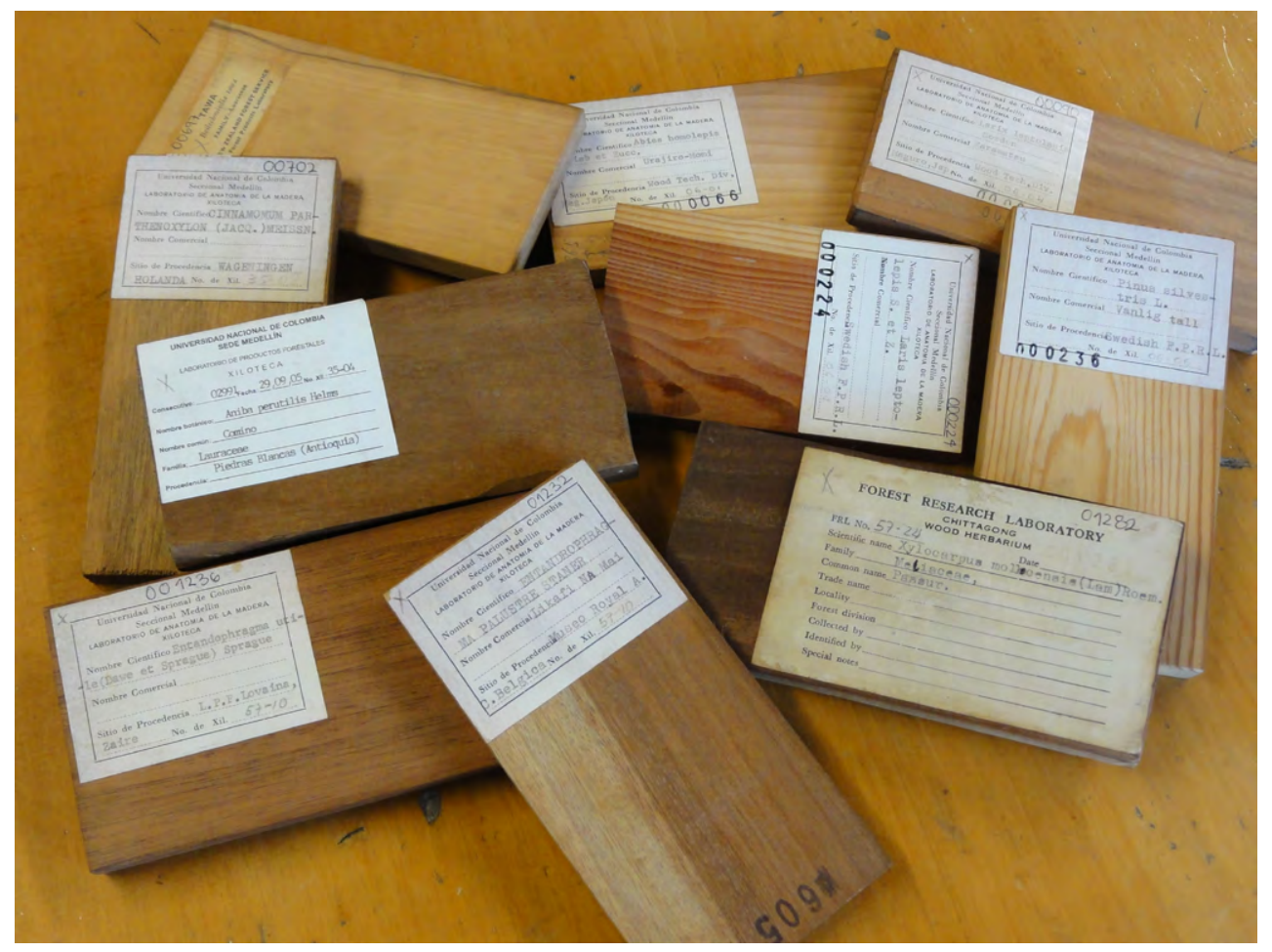

Figura 3. Muestras en forma de pequeñas tabletas en las colecciones de referencia o xilotecas. 
importancia, pues debe ser hecha manteniendo el mismo código de las muestras botánicas y con marcador indeleble o lápiz, que garanticen su permanencia a pesar del alto contenido de humedad en la madera. Las etiquetas plásticas o metálicas no se recomiendan pues con frecuencia se desprenden. Al mismo tiempo que se colecta la muestra, también debe reunirse información adicional sobre la fecha, nombre del colector, dimensiones, forma, fenología y estado fitosanitario del árbol, localidad y características del terreno, altitud y coordenadas y nombre común dado a la especie en la región.

Aunque el alto contenido de humedad que posee la madera en el momento de su corte, puede causar su rápido deterioro, no se recomienda aplicarle ningún producto químico debido a que puede alterar características como el color y olor de las muestras (Lamb \& Curtis, 2005). Lo mejor es transportar los especímenes en costales o cualquier otro material diferente al plástico, con el objetivo de permitir su natural proceso de secado. Si es necesario esperar para su despacho, los especímenes deben ser almacenados en un lugar seco y fresco y nunca directamente expuestas a la radiación solar. Si se empacan en cajas para su envío, se recomienda usar papel periódico para envolverlas de manera individual.

Una vez que las maderas se trasladan a la institución a la cual pertenecerán y han perdido el exceso de humedad, deben someterse a un periodo de cuarentena en una estufa a $60^{\circ} \mathrm{C}$ durante tres días, periodo que garantiza la muerte de huevos o larvas en su interior. Posteriormente, a cada una se le asigna una etiqueta que contiene un número consecutivo o de ingreso a la colección y un código numérico que representa la familia y el género, además de información sobre su nombre común, nombre botánico, familia y procedencia. También se diligencia una ficha de ingreso que incluye toda la información tomada en campo, adicionando el nombre del herbario donde fueron depositadas las muestras botánicas, su estado y número de registro, además del número de ejemplares disponibles para intercambio. Por último, se registra la muestra en la base de datos que contiene toda la información de la xiloteca y se ingresa a la colección.

En el siglo pasado, a pesar de que durante la colecta del material se obtenían también muestras botánicas muchas xilotecas no consignaban el número de registro de las mismas en el herbario correspondiente (Wiedenhoeft, 2014), práctica que tampoco se empleaba en la Xiloteca MEDELw, donde hace pocos años se comenzó a consignar dicha información, de manera que en la base de datos solo el $2 \%$ de los ejemplares cuentan con el número de estos registros en los Herbarios Gabriel Gutiérrez Villegas "Medel" de la Universidad Nacional de Colombia sede Medellín y Gilberto Emilio Mahecha Vega "UDBC" de la Universidad Distrital en Bogotá.

Otras colecciones que han surgido a partir de la xiloteca y que, además de compartir el espacio físico, tienen gran importancia en términos de la labor que cumplen son: la colección Canje o intercambio, que contiene duplicados de las muestras que ingresan a la colección permanente; la colección Muestras de trabajo, que cumple una función inmediata en labores de docencia y extensión y está compuesta por muestras de las maderas más comerciales en Antioquia y el país e identificadas con base en los especímenes de la xiloteca; y la colección Placoteca o microteca con un muestrario de placas permanentes de numerosas especies, utilizadas para realizar su estudio microscópico.

\section{BENEFICIOS DE LAS XILOTECAS}

Como se ha dicho, los beneficios que traen consigo las xilotecas se pueden enumerar de la siguiente manera: se constituyen como importantes reservorios biológicos que pueden albergar duplicados de especímenes valiosos, cuya seguridad aumenta al estar depositados en al menos dos colecciones; permiten desarrollar estudios de descripción e identificación de especies que frecuentemente comienzan con características macroscópicas ampliamente utilizadas en trabajos de campo a nivel 
nacional e internacional, particularmente adecuadas para realizar una determinación básica que puede arrojar respuestas concluyentes o solo reducir el número de posibles géneros o especies a los cuales pertenece el ejemplar (Mainieri et al., 1983; Loureiro et al., 1994; Vásquez \& Ramírez, 2005), y que posteriormente consideran una identificación microscópica con base en un mayor número de estructuras (Wheeler et al., 1989; García et al., 2002; Richter et al., 2004; White \& Gasson, 2008); además, permiten la preparación y montaje de bloques de madera y placas permanentes y la realización de intercambios y préstamos de muestras. Por supuesto, estos no son los únicos objetivos que cumple una xiloteca.

Como lo señalan Cornish et al. (2014), en la última década los usuarios de las colecciones de madera ya no son exclusivamente anatomistas y las técnicas de análisis que utilizan son cada vez más refinadas, pues sus ejemplares sirven como fuente para análisis de ADN e isótopos estables en una variedad de investigaciones que pueden ayudar a rastrear el origen de los árboles talados ilegalmente. Son también una fuente potencial de numerosos compuestos químicos cuya variación es la base que utiliza la espectroscopia infrarroja para separar maderas similares y que, de otra forma, requerirían el muestreo de árboles in situ para su observación y determinación (Kagawa \& Leavitt, 2009; Braga et al., 2011; Wiedenhoeft, 2014), agregando que los especímenes de madera también son utilizados por investigadores en las artes y humanidades, sirven para contar la historia de las colecciones y para la realización de exhibiciones regulares en muchos contextos y para toda clase de espectadores.

Adicionalmente, tanto los estudios en tecnología de la madera como aquellos relacionados con su comercialización y mercadeo tienen un fuerte apoyo en el material correctamente identificado y disponible en las xilotecas, que representa especies cuyo aprovechamiento es autorizado o restringido, y contribuye enormemente en el cumplimiento de las leyes o reglamentos que rigen el manejo forestal de los países en procura de la sostenibilidad y la conservación, al confirmar la legalidad de la madera extraída de sus bosques (Gasson, 2011; Johnson \& Laestadius, 2011; Gasson et al., 2011). Con este mismo propósito, la información contenida en sus muestras se usa para desarrollar aplicaciones para dispositivos móviles que apoyan el monitoreo y control de la comercialización de maderas (Koch et al., 2011; Sarmiento et al., 2011; López et al., 2016).

Aunque ya en la Roma del primer siglo a.C., Plinio el Viejo, en su obra Historia Naturalis, describe por primera vez elementos de la anatomía de la madera como albura y duramen, aceites esenciales, fibras y nudos, entre otros (García et al., 2003). Así, tanto el estudio de la morfología, adaptaciones ecológicas y variación de la madera (Carlquist, 2001), así como su descripción e identificación anatómica, son herramientas que proveen una considerable cantidad de información para su conocimiento, utilización (Mainieri et al., 1983; Mainieri \& Chimelo, 1989; Chichignoud et al., 1990; Acevedo \& Kikata, 1994; Vásquez \& Ramírez, 2005), diferenciación, trazabilidad, certificación, control de comercialización, protección de especies muy similares (García et al., 2002; Coradin et al., 2009; Vásquez et al., 2010; Bernal, et al., 2011) y en estudios para la medición de rasgos funcionales (Salgado et al., 2016), son herramientas aun pobremente usadas y valoradas en el país.

De igual forma, aunque la existencia, el tamaño, la variedad y la fortaleza de las xilotecas y del personal especializado en morfología y anatomía de la madera (Cornish et al., 2014) son los pilares fundamentales para el conocimiento e identificación de nuestras especies, también son aspectos poco apreciados y apoyados en Colombia, Ilegando a abordarse en muchas oportunidades de manera muy informal. Como lo expresa Wiedenhoeft (2014), el apoyo de las colecciones de referencia a los planes de manejo sostenible y de conservación puede ayudar a aumentar la conciencia y reconocimiento de sus beneficios científicos y sociales. Así mismo, ayudan a justificar el gasto y esfuerzo invertidos en su establecimiento y manutención, agregando que, históricamente y a nivel mundial, 
la mayoría de las xilotecas han estado activas solo en la medida en que han sido protegidas por uno o más investigadores en una institución, situación que no es ajena a la realidad colombiana.

\section{CONCLUSIONES}

Para terminar, es importante mencionar las necesidades inmediatas y más apremiantes de estas colecciones de referencia en Colombia. Estas son las siguientes:

Su apropiación por parte de las instituciones que garanticen que su existencia no solo esté ligada al nombre y permanencia de un investigador en particular, además de su protección y enriquecimiento, pues a pesar de nuestra amplia diversidad de especies las dos únicas xilotecas registradas que pueden dar cuenta de un riguroso proceso de curaduría y conservación de sus muestras en el país poseen un número de ejemplares muy reducido.

Difundir la existencia e importancia de estas colecciones como legado cultural y científico de la nación. En la actualidad, ni el público ni la comunidad científica conocen la importancia y utilidad de los especímenes que albergan y persiste, además, un errado concepto y enorme confusión sobre la diferencia entre una colección de maderas y una xiloteca.

Realizar un trabajo conjunto entre las xilotecas de las diferentes instituciones para fortalecer sus vínculos, efectuar intercambio de muestras e información y garantizar que las futuras generaciones puedan recibir el legado y comprender su valor, fomentando de paso la conformación de otras colecciones y la formación de otros profesionales en el área.

Consolidar de manera urgente una política de adquisición de ejemplares en acuerdo con los diferentes herbarios del país, que contribuya al crecimiento y mejora de los registros de los especímenes para que representen de manera más completa nuestra riqueza natural.

\section{CONFLICTO DE INTERESES}

El autor declara no tener conflicto de intereses.

\section{CONTRIBUCIÓN POR AUTOR}

El autor único es responsable de la obra en todos los aspectos que condujeron a la elaboración de su publicación.

\section{REFERENCIAS BIBLIOGRÁFICAS}

Acevedo, M. \& Y. Kikata. (1994). Atlas de maderas del Perú. Lima: Universidad Nacional Agraria La Molina Perú-Universidad de Nagoya Japón. 202 p.

Bernal, R., Coradin, V., Camargos J., Costa, C. \& Pissarra, J. (2011). Wood anatomy of Lecythidaceae species called "Tauari". IAWA Journal, 32(1), 97-112.

Braga, J., Pastore, T., Coradin, V., Camargos, J. \& Silva, A. (2011). The use of near infrared spectroscopy to identify solid wood specimens of Swietenia macrophylla (CITES Appendix II). IAWA Journal, 32(2), 285-296.

Carlquist, S. (2001). Comparative wood anatomy. Systematic, ecological, and evolucionary aspects of dicotyledon wood. Heidelberg (Berlin): Springer Verlag. 435 p.

Chichignoud, M., Déon, G., Détienne, P., Parant, B. \& Vantome, P. (1990). Atlas de maderas tropicales de América Latina. Traducción: Isabel Bagué Bofia. Yokohama (Japón): Organización Internacional de las Maderas Tropicales-Centre Technique Forestier Tropical. $217 \mathrm{p}$.

Coradin, V., Alves C., Marques, L. \& Rangel, E. (2009). Madeiras similares ao mogno (Swietenia macrophylla King): Chave ilustrada para identificação em campo. Brasília: Serviço Florestal Brasileiro/ LPF. 28 p.

Cornish, C., Gasson, P. \& Nesbitt, M. (2014). The Wood Collection (Xylarium) of the Royal Botanic Gardens, Kew. IAWA Journal, 35(1), 85-104. 
García, L., de Palacios, P., Guindeo, A., García, L., Lázaro, I., González, L., Rodríguez, Y., Bobadilla, I., Fernández, F. \& Camacho, A. (2002). Anatomía e identificación de maderas de coníferas a nivel de especie. Madrid: Fundación Conde del Valle de Salazar. 421 p.

García, L., Guindeo, A., Peraza, C. \& de Palacios, P. (2003). La madera y su anatomía: anomalías y defectos, estructura microscópica de coníferas y frondosas, identificación de maderas, descripción de especies y pared celular. Madrid: Fundación Conde del valle de Salazar. 327 p.

Gasson, P. (2011). How precise can wood identification be? Wood anatomy's role in support of the legal timber trade, especially CITES. IAWA Journal, 32 (2), 137-154.

Gasson, P., Baas, P. \& Wheeler, E. (2011). Wood anatomy of Cites-listed tree species. IAWA Journal, 32 (2), 155-198.

Hoadley, B. (1990). Identifying wood: Accurate results with simple tools. Connecticut: The Taunton Press Inc. 223 p.

Johnson, A. \& Laestadius, L. (2011). New laws, new needs: The role of wood science in global policy efforts to reduce illegal logging and associated trade. IAWA Journal, 32(2), 125-136.

Kagawa, A. \& Leavitt, S. (2009). Stable carbon isotopes of tree rings as a tool to pinpoint the geographic origin of timber. Journal of Wood Science, 56, 175-183.

Koch, G., Richter, H. \& Schmitt, U. (2011). Design and application of CITESwoodID, computer-aided identification and description of CITES-protected timbers. IAWA Journal, 32(2), 213-220.

Lamb, H. \& Curtis, A. (2005). A Guide for developing a wood collection. Madison: Forest Products Society. $56 \mathrm{p}$.

León, H. (2009). 50 años de la xiloteca MERw, patrimonio científico de Venezuela (1959-2009). Pittieria, 33, 111-120.

López, R., Pulido, N., Nieto, J. \& Moreno, J. (2016). Especies maderables versión 2. Catálogo de especies maderables de Colombia. Bogotá: Laboratorio de
Tecnología de Maderas José Anatolio Lastra, Universidad Distrital Francisco José de Caldas.

Loureiro, A., de Freitas, J. \& Castro e Silva, A. (1994). Chave para identificação macroscópica de madeiras da Amazônia. Manaus: Secretaria de Estado do Meio Ambiente-Ciência e Tecnologia do Estado do Amazonas. 71 p.

Lynch, A. \& Gasson, P. (2010). Index Xylariorum. Edition 4. London: Royal Botanic Gardens. Recuperado de: www.kew.org/collections/ wood-index/ Index_Xylariorum4.htm.

Mainieri, C. \& Chimelo, J. (1989). Fichas de características das madeiras brasileiras. São Paulo (Brasil): Instituto de Pesquisas Tecnológicas do Estado de São Paulo, Divisão de madeiras. 418 p.

Mainieri, C., Chimelo, J. \& Alfonso, V. (1983). Manual de identificação das principais madeiras comerciais brasileiras. São Paulo: Instituto de Pesquisas Tecnológicas do Estado de São Paulo, Divisão de madeiras. 241 p.

Richter, H., Grosser, D, Heinz, I. \& Gasson, P. (eds.). (2004). IAWA List of microscopic features for softwood identification. IAWA Journal, 25(1), 1-70.

Salgado, B., Pulido, E., Cabrera M., Ruíz, C. \& Paz, H. (2016). Protocolo para la medición de rasgos funcionales en plantas. En B. Salgado (ed.). La ecología funcional como aproximación al estudio, manejo y conservación de la biodiversidad: protocolos y aplicaciones (pp. 36-79). Bogotá: Instituto de Investigación de Recursos Biológicos Alexander von Humboldt.

Sarmiento, C., Détienne, P., Heinz, J., Molino, F., Grard, P. \& Bonnet, P. (2011). PI@ntWood: a computer-assisted identification tool for 110 species of amazon trees based on wood anatomical features. IAWA Journal, 32(2), 221-232.

Stern, W. (1957). Guide to institutional wood collections. Tropical Woods, 106, 1-29.

Stern, W. (1988). Index xylariorum. Institutional wood collections of the world 3. IAWA Bulletin, 9, 203-252.

Vásquez, A. \& Ramírez, A. (2005). Maderas Comerciales en el Valle de Aburrá. Medellín: Área Metropolitana del Valle de Aburrá. 246 p. 
Vásquez, A., Alcántara, E. \& Herrera, A. (2010). Wood anatomy of Colombian Podocarpaceae (Podocarpus, Prumnopitys and Retrophyllum). Botanical Journal of the Linnean Society, (164), 293-302.

Wheeler, E., Baas, P. \& Gasson, P. (eds.). (1989). List of microscopic features for hardwood identification with an Appendix on non-anatomical information. IAWA Bulletin, 10(3), 219-332.
White L. \& Gasson, P. (2008). Mahogany. Richmond (UK): Royal Botanic Gardens, Kew. 99 p.

Wiedenhoeft, A. (2014). Curating Xylaria, En: J. Salick, K. Konchar \& Ma. Nesbitt (eds.). Chapter 9. Curating biocultural collections: A handbook (pp. 127134). Richmond: Royal Botanic Gardens, Kew.

\section{(c) (1) $(\Theta$

